\title{
An Efficient Image Segmentation Technique by Integrating FELICM with Negative Selection Algorithm
}

\author{
${ }^{1}$ Er. Pratibha Thakur and ${ }^{2}$ Er. Sanjeev Dhiman \\ ${ }^{1}$ Research Scholar, ${ }^{2}$ Assistant Professor, Department of Computer Science and \\ Engineering DAV University, Jalandhar, Punjab, India \\ 'pratibhathakur70@gmail.com, ${ }^{2}$ sanhamir@gmail.com
}

\begin{abstract}
Segmentation is a efficient technique of dividing the image into different regions or segments. Most of the researchers took clustering as the best method of segmenting an image. In clustering we try to increase the similarity within a same class and decrease the similarity between the classes. Many clustering algorithms were developed like FCM, FLICM and FELICM which are considered as the best algorithms to cluster the data. In our paper, we combine FELICM (Fuzzy Edge and Local Information C-Mean) with the negative selection algorithm. Negative selection algorithm is an evolutionary method which is based on artificial immune systems. The proposed method result shows us high accuracy results and even solves the problem of over segmentation.
\end{abstract}

Keywords: FCM, FLICM, FELICM, Negative Selection Algorithm

\section{Introduction}

Image Segmentation is a critical methodology of image transforming and comprehension in digital image processing. This technique is basically used for separating the image into different parts of homogeneity. The motive of image division is to enhance the representation of a picture into something that is more important. The basic use of it is to find the location of objects, boundaries, lines etc. in the digital images. Clustering is a way in which a data set or say pixels are interchanged by groups, pixels may place together because of the same color, composition etc.

There are number of clustering techniques available which are used to segment the image in distinct ways. Out of various techniques available some of the important ones are following: k-mean clustering, Fuzzy C-Mean clustering(FCM),Fuzzy c-mean with local information(FLICM), Fuzzy edge and local information c-mean(FELICM).

K-Mean clustering can also be heard as hard clustering. The neighboring pixels of an image are having almost similar i.e. they are approximately same. So we choose Euclidean distance as a factor to cluster the pixels. Partition of an image into clusters are done with an iterative technique from which the choice of $\mathrm{k}$ cluster centers, randomly then every pixel in the image is allocated to the unique cluster. The cluster centre is reevaluated by averaging all of the pixels in the centre's c-mean shows problems when distinct clusters have different size, shape and density [1].Fuzzy clustering is also called soft segmentation. It uses the methodology of partial membership of belonging explained by a membership function [2].In this every point has a degree of belonging of clustering instead of each pixel does belong to one cluster only so it also removed the flaws in the $\mathrm{k}$ mean. After it the new technique fuzzy local information c-mean (FLICM) was introduced. It improved the clustering performance when compared with the fuzzy cmeans and meantime. In this the main idea on which it works is that it uses fuzzy local (both spatial and gray level) similarity measure, planning to ensure noise insensitiveness and image information preservation [3]. To remove the noise effects spatial relationship between pixels can be used but when we use it most of the times it produces boundary 
zones when there are mixed pixels around the edges. To overcome from this problem we have an another technique called fuzzy C-means with edge and local information (FELICM) which is based upon image spatial clustering method that lessens the edge degradation by including the weights of pixels within nearby neighbor's windows [4].Fuzzy c-means (FCM) with edge and local information (FELICM) also provides the he more edge exactness when compared with the FLICM [11].

In our paper, we introduce a new integrated Negative selection based FELICM approach to improve the accuracy of the segmentation procedure further. The inspiration driving the proposed methodology is basic and powerful. NSA is based on artificial immune systems. The NSA was first utilized by Forrest. It is a standout amongst the most popular models that influence the improvement of the existing artificial immune systems. The use of negative selection technique will allow finding an optimistic, adaptive threshold to segment the image in a more efficient manner. Negative selection based algorithms have been used in different application areas, such as anomaly detection. Forrest (1994) proposed a negative selection algorithm. The main idea behind the algorithm is to produce a set of detectors. Firstly, candidates are generated randomly and then those that recognize training self-data are discarded, and these detectors can later be used to detect anomaly [5]. A set of self elements is defined within that shape space and a set of detectors is derived from them, which decides whether an incoming new feature data from the dynamic process is normal (self) or not (non-self). Thus, in this way, the NSA does its main task of discrimination between self and nonself [9].

\section{Proposed Methodology}

We have analyzed from the previous methods that there are still some gaps like with the blended area have not been considered and the impact of color have been disregarded [10]. Also, there exist some isolated regions which FELICM was unable to remove properly. So to overcome these issues we have proposed a new method in which we integrated two different algorithms i.e. FELICM and Negative Selection Algorithm. Figure 1: is representing the flow of the proposed methodology.

The explanation of the proposed algorithm is given below:

STEP 1: Take the image as an input. It could be either grey level image or color image.

STEP 2: After this FELICM and NSA are applied on the same image separately.

\section{A. APPLY FELICM}

\section{(a) Segmentation using FELICM Algorithm}

- First of all get the principle component analysis which is mathematical procedure that uses the orthogonal transformation. In this image in RGB form converted into PCA and then each component converted into vectors.

They can be concatenated using equation.

$$
I I V=\operatorname{cat}\left(\frac{\alpha}{2}\right.
$$

is input image vector 


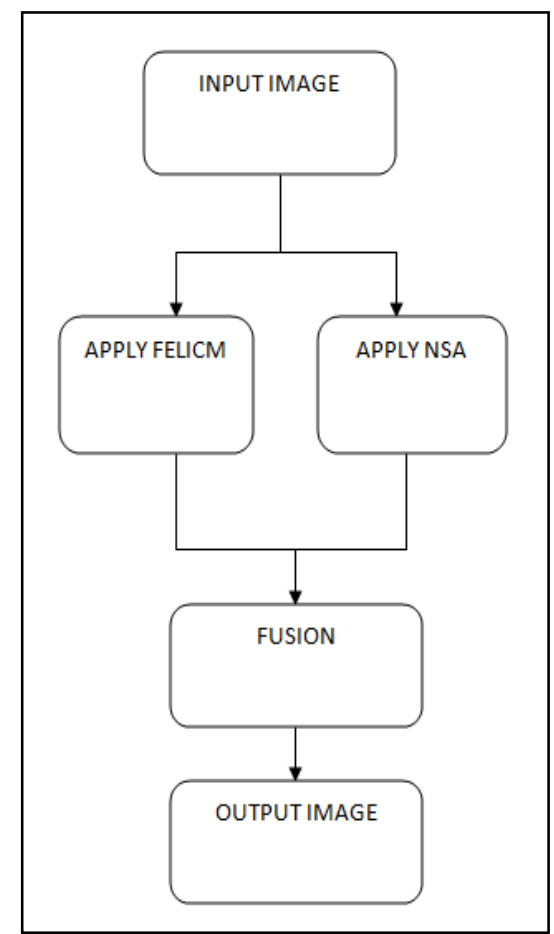

Figure 1. Proposed Algorithm

- Principle component is used for calculating Eigen Values using the following equation where VV represents vector values.

$$
V V=\text { princomp }(I I V)
$$

- To get PCA Vector using Vector values the following equation can be used.

- $\quad$ PCA image can be obtained using the following.

$$
\text { Vector }=V V_{\text {a }}
$$

$$
\text { OVI }=I I V * \text { Vector }
$$

$O V I$ is output image vector

- Edges are extracted using the canny edge operator along with the information obtained using PCA and otsu method.

- Then FELICM based clustering is done in which the objective function can be defined as

$$
J=\sum_{i=1}^{N} \sum_{K=1}^{C}[Q k i+G k i]
$$

\section{B. APPLY NSA}

STEP 3. Now fusion will be done on two different images got from the FELICM and NSA.

STEP 4. All pixels of the picture will be allotted to their closest groups and proposed segmentation method will be attained.

STEP 5. After obtaining the results color labeling will be done.

STEP 6. Output of the proposed method is obtained with the improved accuracy.

\section{Experimental Results}

The main title (on the first page) should begin $13 / 16$ inches ( 7 picas) from the top edge of the page, centered, and in Times New Roman 14-point, boldface type. Capitalize the first letter of nouns, pronouns, verbs, adjectives, and adverbs; do not capitalize articles, 
coordinate conjunctions, or prepositions (unless the title begins with such a word). Please initially capitalize only the first word in other titles, including section titles and first, second, and third-order headings (for example, "Titles and headings" — as in these guidelines). Leave two blank lines after the title.

We implemented the proposed algorithm on a window 7 PC with an Intel ${ }^{\circledR}$ Core (TM) i5-2430M CPU @ 2.4GHz processor, running MATLAB R2012b. we categorize the experimental images into building images containing original image, FELICM images and Proposed algorithm images. We have tested our proposed algorithm on various images and the results for the same are shown in the Table 1

Table 1. Matlab Inbuilt Images Results

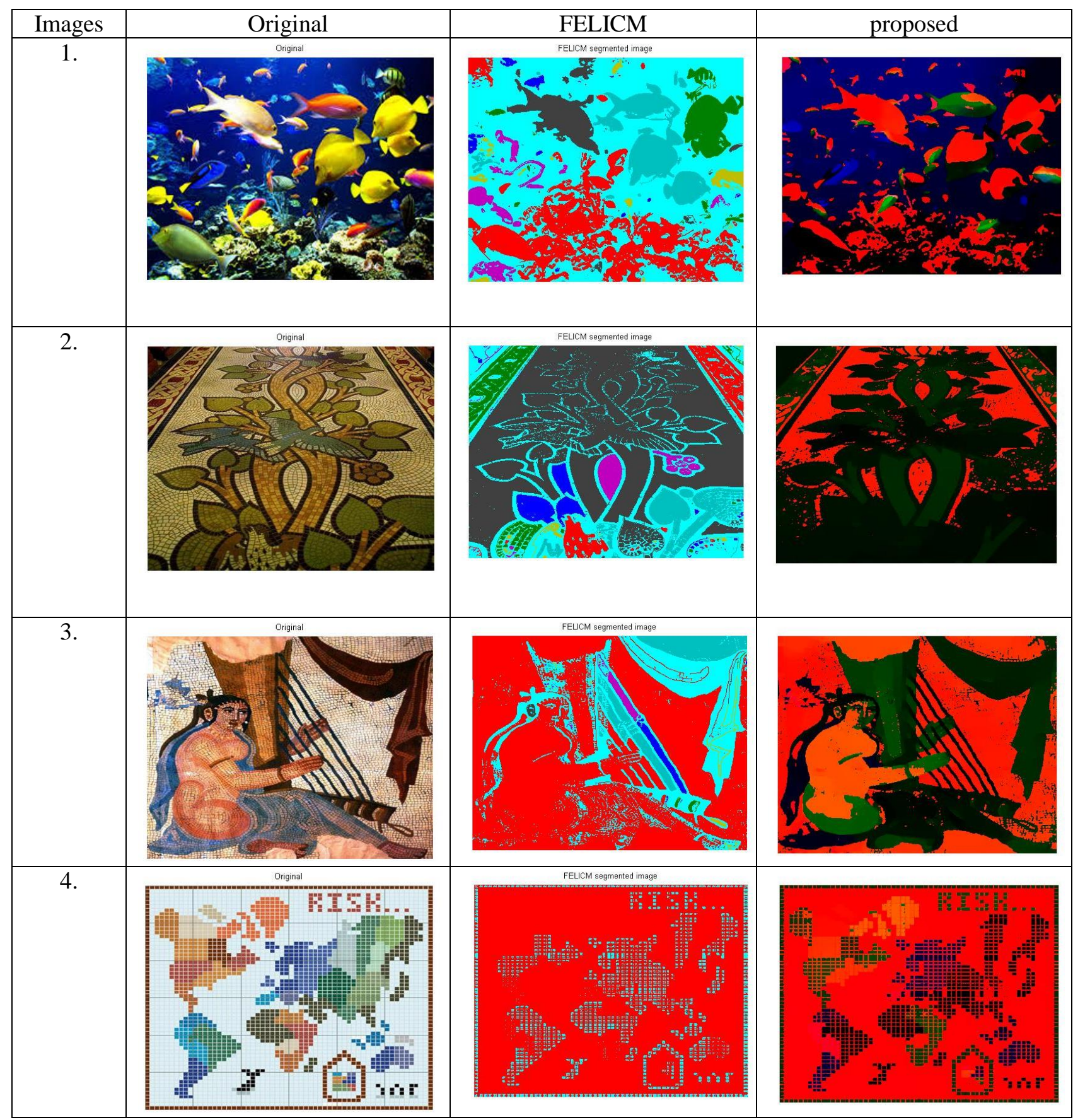




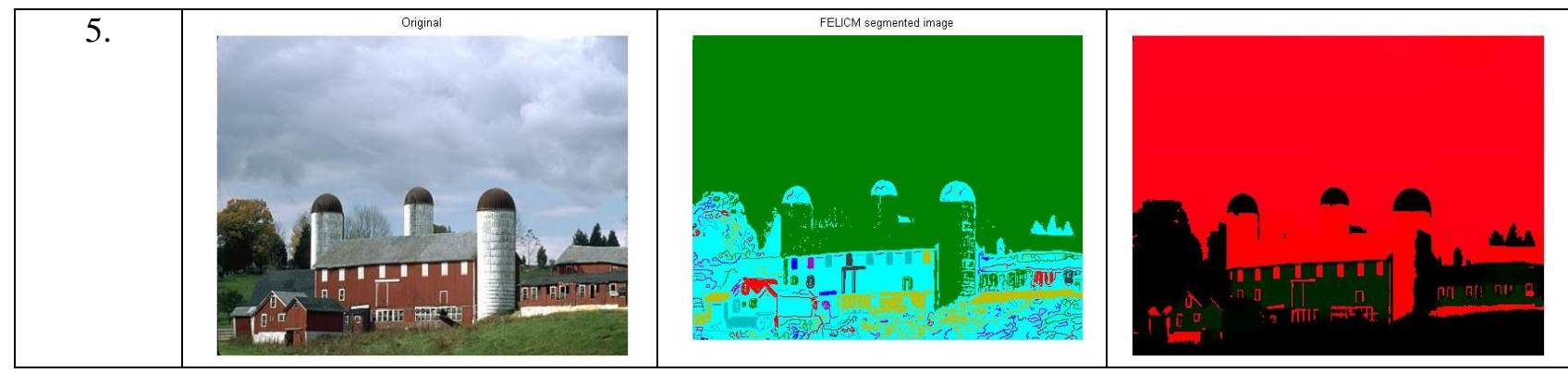

\section{Performance Evaluation}

We have tested our proposed methodology on the inbuilt MATLAB images of car, flower, butterfly, turtle and octopus. We have analyzed that our proposed algorithm is giving us the three parameters with the improved performance when compared with the previous algorithm. These three parameters are PSNR value, accuracy and entropy. As PSNR, Accuracy and entropy need to be maximize therefore proposed algorithm is showing better results than the previous methods.

$$
\begin{aligned}
& \text { MSE }=\frac{1}{M N} \sum_{i=0}^{M-1} \sum_{j=0}^{M-1}\left(f_{i j}-f_{i j}^{\prime}\right)^{2} \\
& 0 \leq i \leq M-1,0 \leq j \leq N-1 \\
& \text { PSNR }=10 \log \frac{\left(2^{n}-1\right)}{M S E}
\end{aligned}
$$

Accuracy_Accuracy is measured that how close you are to the actual value. For this we need to calculate the error.

$$
\% \text { Error }=\frac{(Y V-A D) \times 100}{A V}
$$

Where: YV is your measured Value \& AV is the Accepted Value.

Entropy gives us the amount of information that is carried by an image.

Table 2. Qualitative Analysis

\begin{tabular}{|l|l|l|l|l|l|l|l|l|l|}
\hline \multirow{2}{*}{ Images } & \multicolumn{9}{|l|}{ PSNR } \\
\cline { 2 - 10 } & FCM & FELICM & Proposed & FCM & FELICM & Proposed & FCM & FELICM & Proposed \\
\hline 1 & 34.8794 & 59.5368 & 71.9378 & 0.1509 & 0.8302 & 0.9661 & 0.7102 & 7.6697 & 14.7039 \\
\hline 2 & 31.5645 & 63.6417 & 68.2775 & 0.5128 & 0.6708 & 0.8820 & 3.1233 & 4.8258 & 9.2805 \\
\hline 3 & 31.2604 & 64.4310 & 79.1339 & 0.5503 & 0.6257 & 0.9801 & 3.4707 & 4.2681 & 17.0035 \\
\hline 4 & 30.8129 & 58.6155 & 66.0360 & 0.5391 & 0.8457 & 0.8422 & 3.3642 & 8.1150 & 8.0195 \\
\hline 5 & 35.4205 & 56.8779 & 75.9767 & 0.0781 & 0.4705 & 0.9898 & 0.3533 & 2.7615 & 19.8938 \\
\hline 6 & 33.1903 & 56.1610 & 62.6521 & 0.2768 & 0.7697 & 0.8338 & 1.4072 & 6.3779 & 7.7948 \\
\hline 7 & 33.0539 & 64.0907 & 72.1904 & 0.2615 & 0.5143 & 0.9794 & 1.3168 & 3.1360 & 16.8540 \\
\hline 8 & 33.2940 & 58.9451 & 67.8136 & 0.2275 & 0.4837 & 0.9404 & 1.1211 & 2.8710 & 12.2466 \\
\hline 9 & 31.1907 & 57.6239 & 73.6801 & 0.4327 & 0.8130 & 0.9287 & 2.4615 & 7.2827 & 11.4678 \\
\hline 10 & 32.5380 & 57.2507 & 71.8920 & 0.3420 & 0.7327 & 0.8790 & 1.8177 & 5.7308 & 9.1718 \\
\hline
\end{tabular}




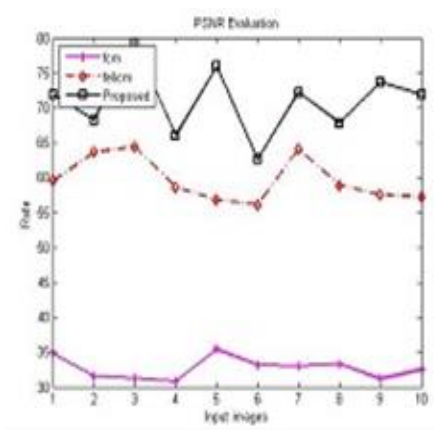

(a)

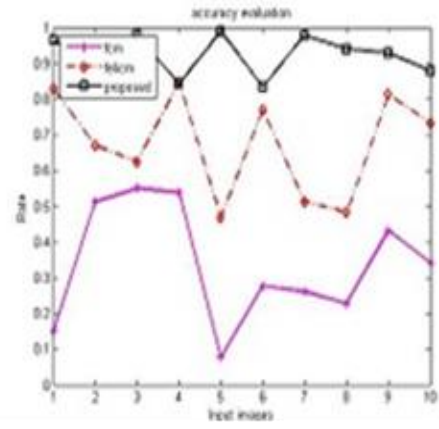

(b)

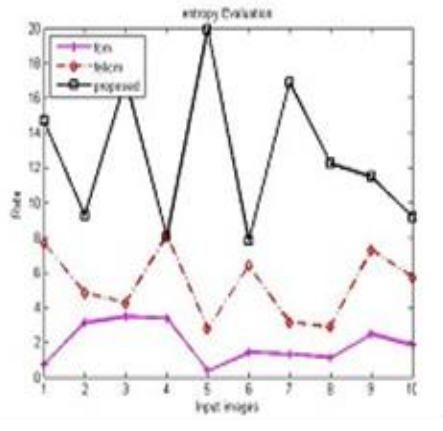

(c)

\section{Figure 3. (a) Graph for PSNR, (b) Graph for Accuracy and (c) Graph for Entropy}

\section{Conclusion}

The previous clustering method gives the isolated samples of pixels so mostly it results in isolated regions. Traditional clustering methods are unable to remove isolated regions. FLICM produces boundary zones. FELICM somehow eliminates these problems, but the proposed method gives very efficient result as compared to previous clustering methods. Moreover, it also eliminates the over segmentation which can be clearly seen in the Matlab inbuilt images result. In our proposed methodology when FELICM is integrated with the negative selection algorithm, the three of the parameters i.e. PSNR value, accuracy and entropy are showing the improved and better results when it is compared with the existing FELICM.

\section{References}

[1] K. Shrivastava, N. Gupta and N. Sharma, "Survey paper on image segmentation using k-means clustering”, International Journal of Advanced Technology \& Engineering Research (IJATER) vol. 4no. 5, (2014) September.

[2] Y. Yang, "Image segmentation by fuzzy c-means Clustering algorithm with a novel Penalty term", computing and informatics, vol. 26, (2007), pp. 17-31.

[3] S. Krinidis and V. Chatzis, "A Robust Fuzzy Local Information C-Means Clustering Algorithm”, ieee transactions on image processing, vol. 19, no. 5, (2010) May.

[4] N. Li, H. Huo, Y.-m. Zhao, X. Chen and T. Fang, "a spatial clustering method with edge weighting for image segmentation", IEEE geoscience and remote sensing letters, vol. 10, no. 5, (2013) September.

[5] J. R. Al-Enezi, M. F. Abbod and S. Alsharhan, "Artificial immune systems - models, algorithms and applications" IJRRAS, vol. 3, no. 2, (2010) May.

[6] A. Halder, S. Pramanik and A. Kar, "Dynamic Image Segmentation using Fuzzy C-Meansbased Genetic Algorithm", IJCA vol. 28, no. 6, (2011) August.

[7] E. Bendiab and M. K. Kholladi, "Unsupervised Classification Based Negative Selection Algorithm".

[8] P. K. Mahapatra, M. Kaur, S. Sethi, R. Thareja, A. Kumar and S. Devi, "Improved thresholding based on negative selection algorithm (NSA)", Springer-Verlag Berlin Heidelberg (2013).

[9] P. K. Mahapatra, S. Ganguli and A. Kumar, "A hybrid particle swarm optimization and artificial immune system algorithm for image enhancement”, Springer-Verlag Berlin Heidelberg (2014).

[10] A. K. Randhawa and R. Mahajan, "Modified Clustering Based Image Segmentation", International Journal of Advanced Research in Computer Engineering \& Technology (IJARCET) vol. 3, no. 8, (2014) August.

[11] Rilanthiraiyan and V. M. Navaneethakrishnan "Spatial clustering method for satellite image segmentation", iosr journal of electronics and communication engineering (iosr-jece) E-ISSN: 22782834, P-ISSN: 2278-8735, vol. 9, no. 2, ver. III (2014) March-April, pp. 67-73.

[12] M. Xess and S. A. Agnes, "Survey on Clustering Based Color Image Segmentation and Novel Approaches to Fcm Algorithm", IJRET, eISSN: 2319-1163.

[13] Ramya and Jemimah Simon, "Image Segmentation Using FELICM Clustering Method", (IJERA) ISSN: 2248-9622, (2014) March 1.

[14] S. Kaur and V. K. Banga, "Content Based Image Retrieval: Survey and Comparison between RGB and HSV model”, (IJETT), vol. 4, no. 4, (2013) April. 


\section{Authors}

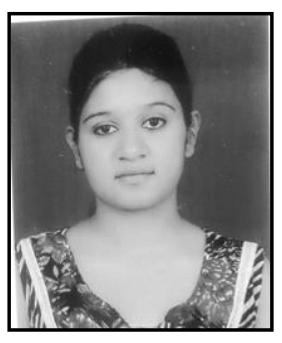

Er. Pratibha Thakur, she is pursuing, M. Tech, CSE Department DAV University, Jalandhar Punjab. She completed B. Tech from BCET Gurdaspur, PTU Jalandhar. She had many projects on $\mathrm{C}++$, ASP.NET and Java. She also published many papers in international journals.

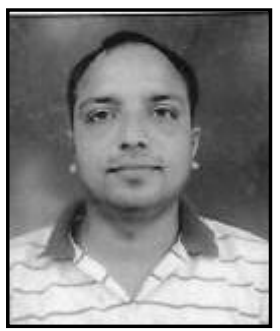

Er. Sanjeev Dhiman, he is working as Assistant professor in the Department of Computer Science and Engineering at DAV University, Jalandhar Punjab (INDIA). His research areas include Digital Image Processing and Data Mining. He has various research publications in various international journals and has also presented several papers in various national and international conferences. 
International Journal of Signal Processing, Image Processing and Pattern Recognition Vol.8, No.10 (2015) 